ROCKY MOUNTAIN

JOURNAL OF MATHEMATICS

Volume 7, Number 1, Winter 1977

\title{
INFINITE GROUPS WITH NORMALITY CONDITIONS ON INFINITE SUBGROUPS
}

\author{
RICHARD E. PHILLIPS
}

I. Introduction. In the papers [2, $\$ \$ 2$ and 3], and [3] S. N. Cernikov studies infinite groups $G$ with the property that every infinite subgroup $H$ of $G, H \neq G$, is distinct from its normalizer in $G$. Groups with this property are called IN groups. Another way of stating the $I N$ property is "every infinite subgroup of $G$ is ascendant in $G$ ".

IN groups are of two types:

(i) $\mathrm{N}$-groups (i.e. groups with the normalizer condition) or (ii) $I N$ groups with a self normalizing (necessarily finite) proper subgroup.

Obviously, the classes (i) and (ii) exhaust all IN groups. In [2, Theorems 2.1 and 2.2], Cernikov gives a detailed description of the structure of locally finite groups of type (ii). In the later paper [3], it is shown that non-torsion $I N$ groups are $N$ groups.

In this paper, we give an exposition of these results. Our proofs are quite different and, in most cases, considerably shorter than those given in [2] and [3]. The papers [2] and [3], especially [2], make use of several results not easily accessible to non-Russian mathematicians. We have attempted here to use techniques that, for the most part, can be found in the books [14], or [18] .

Also, we have put the results of [2] in a somewhat wider context. This has been achieved by replacing the notion of an ascendant subgroup by a serial subgroup. Thus, Cernikov's Theorems 2.2 and 3.4 of [2] are consequences of our Theorem D ( $\$$ III). We also indicate how to construct groups satisfying Cernikov's structure theorem for groups of type (ii) (Theorem $\mathrm{F}$ in $\S \mathrm{V}$ ).

Finally in $\S \mathrm{VI}$, we give a shorter proof of the results of [3], and note some related problems that remain unsolved.

II. Notation on Basic Concepts. The class of nilpotent groups is denoted by $\Re$, while the class of finite groups is given by $\mathfrak{\mho}$. If $\Sigma$ is any class of groups, $L \Sigma$ is the class of groups having a local system of $\Sigma$ subgroups [18; p. 5]. Thus $L \Re$ and $L \mathscr{F}$ denote locally nilpotent and locally finite groups respectively.

$H$ is a serial subgroup of $G(H \operatorname{ser} G)$ if there is a series of $G$ that contains $H$ as a member [18; p. 9]. The notions of ascendant $(H$ asc $G)$,

Received by the editors on June 10, 1974 and in revised form on August 28, 1974. 
descendant $(H$ desc $G)$ and subnormal $(H$ sn $G)$ subgroups are specializations of serial subgroups. We let $\mathfrak{R}(s), \mathfrak{R}(a), \mathfrak{R}(d), \mathfrak{N}(\mathrm{sn})$ denote the classes of all groups in which all subgroups are respectively serial, ascendant, descendant or subnormal. Similarly, $I \mathfrak{N}(s), I \mathfrak{I}(a), I \mathfrak{N}(d)$, and $I \Re(s n)$ denote the classes of infinite groups in which all infinite subgroups are serial, ascendant, descendant or subnormal. We note that several of the above classes are denoted by other symbols in the literature; e.g. $\mathfrak{N}(s)=\tilde{N}[14 ;$ p. 221$], \mathfrak{R}(a)=N=$ normalizer condition $\left[14 ;\right.$ p. 220], $\Re(s n)=N_{0}$, [15; p. 319].

2.1. Serial Subgroups. The following characterization of serial subgroups in $L \mathscr{z}$ groups plays an essential role in what follows.

2.1.1 [11] or [16]. If $G \in L \mathfrak{z}, H$ ser $G$ if and only if for every finite subgroup $L$ of $G, H \cap L \operatorname{sn} L$.

We call a class $\Sigma$ of groups a radical class if

(i) $L(\Sigma) \subset \Sigma$,

(ii) subgroups of $\Sigma$ groups are $\Sigma$ groups,

(iii) in any group, the join $\Sigma(G)$ of all the normal $\Sigma$ subgroups of $G$ is a $\Sigma$ group.

The following fact is implicit in the work of Plotkin [17], and is not difficult to prove directly.

2.1.2. If $\Sigma$ is a radical class, $G \in L \mathscr{z}$ and $H$ is a serial $\Sigma$ subgroup of $G$, then $H^{G} \in \Sigma$.

Classes $\Sigma$ for which we use 2.1.2. include $\Pi$ groups, $\Pi$ a set of primes, and $L \Re$ groups.

2.2. Cernikov Groups. A group $G$ is a Cernikov group (or an extremal group) if $G$ is a finite extension of an abelian group with the minimum condition on subgroups $(=\min )$.

If $G$ is an infinite Cernikov group and $d(G)$ is the intersection of the subgroups of finite index in $G$, then $d(G)$ has finite index in $G$ [14; p. 230 ], and $d(G)$ is a periodic divisible abelian group with min (i.e., $d(\mathrm{G})$ is a direct sum of a finite number of quasi-cyclic groups).

If $H$ is an arbitrary abelian group, $p$ a prime and $n$ a positive integer, $H\left(p^{n}\right)$ denotes the subgroup of $H$ generated by the elements of order $p^{n}$.

We require the following facts relating to Cernikov groups.

2.2.1 [1], or [18; p. 84]. If $Y$ is a periodic group of automorphisms of a Cernikov group, then $Y$ is a Černikov group.

2.2 .2 [18; p. 84]. If $H$ is a divisible abelian group and $F$ is a finite group of automorphisms of $H$ then $H=C_{H}(F)[H, F]$. 
2.2.3 [18; p. 230]. A Černikov $p$-group $G$ is hypercentral. Consequently $G \in \mathfrak{R}(a)$.

2.2.4 [18; p. 69]. If $H$ and $K$ are Černikov groups, and $G$ is an extension of $H$ by $K$, then $G$ is a Cernikov group. Also, (and quite simply) subgroups of Cernikov groups are Černikov groups.

2.3. Some Remarks on Injective Modules. Our presentation here is simplified by appealing to recent results of Hartley and McDougall [12]. These results concern modules for integral group rings of certain groups.

We recall that if the abelian group $M$ is a module for the group $G$, then $M$ may be viewed, in a natural way, as an $R=Z G$ module; here $Z G$ is the integral group ring of $G$. Specifically, let $m \in M, g \in G$ and denote by $m^{g}$ the image of $m$ under $g$. Let $\alpha=\Sigma n_{g} g \in Z G$, where the g's are elements of $G, n_{\mathrm{g}} \in Z$, and all but a finite number of the $n_{\mathrm{g}}$ 's $=$ 0 . We define $m \alpha$ by $m \alpha=\Sigma n_{g}\left(m^{g}\right)$. It is easy to check that this product makes $M$ an $R$ module.

The following is a simplified version of Lemma 2.3 of [12].

2.3.1. Suppose $M$ is an abelian $p$ group ( $p$ a prime) and that $M$ is a module for the finite $p^{\prime}$ group $Q$. Let $R=Z Q$. Then $M$ is an injective $R$ module if and only if $M$ is a divisible group.

We also need.

2.3.2 [12; Lemma 2.1]. Let $R$ be a ring with 1 and $V$ be an $R$ module (unitary). Suppose that $V=\oplus\left\{V_{\lambda} \mid \lambda \in \Lambda\right\}$ (module direct sum) and that $\Lambda$ is finite. Let $\bar{V}$ be an $R$-injective hull of $V$. Then $\bar{V}=\oplus$ $\left\{\bar{V}_{\lambda} \mid \lambda \in \Lambda\right\}$ where $\bar{V}_{\lambda}$ is an injective hull of $V_{\lambda}$.

The necessary definitions and theorems on injective modules may be found in the book [6; pp. 385-392]. In particular, we assume both the existence and the various characterizations of injective hulls of modules.

2.4. A Schur-Zassenhaus Property. The following version of the Schur-Zassenhaus property appears in the early work of Cernikov [4]. A proof is also indicated in $[7 ;$ p. 22]

2.4.1. Suppose $G$ is a $L \mathscr{z}$, locally solvable and countable group. Then for every prime $p$, there is a Sylow $p$ subgroup $P$ and a Sylow $p^{\prime}$ subgroup $Q$ such that $G=P Q$.

We also require the following fact, which is of a much simpler nature. 
2.4.2. Let $G \in L \mathscr{Z}$ have a normal Sylow $p$ subgroup $P$ of finite in dex: then $P$ has a complement $K$. Any such complement is a Sylow $p$ subgroup of $G$. Further, any Sylow $p^{\prime}$ subgroup $L$ of $G$ is conjugate to K.

Proof. Let $T$ be a transversal of $P$ in $G$. Then $H=\langle T\rangle$ is finite anc $G=P H$. Further, $P \cap H$ is a normal Sylow $p$ subgroup of $H$. It nov follows from the Schur-Zassenhaus-Feit-Thompson theorem for finit groups [20; p. 224] that $P \cap H$ has a complement $K$ in $H$. Evidently $K$ is a $p^{\prime}$ group, and $G=P H=P K$. Thus $K$ is a complement of $P$. A easy argument shows that $K$ is a Sylow $p^{\prime}$ subgroup of $G$.

Let $L$ be another Sylow $p^{\prime}$ subgroup of $G$. Then $L P / P \simeq L / L \cap$ $\simeq L$, so that $L$ is finite. Since $G \in L \mathscr{F}, Y=\langle L, K\rangle$ is finite. Ob serve also that $Y$ has the normal Sylow $p$-subgroup $Y \cap P$. Agai using the Schur-Zassenhaus-Feit-Thompson theorem [20; p. 227] $w$ deduce that $L$ and $K$ are conjugate in $Y$.

2.5. A Remark on Locally Solvable Groups. We note the theorer of Mal'cev which states that every locally solvable group is an SI group This result is proved in the book $[19 ;$ p. 97] and also in $[14 ;$ p. 183] From this and the Corollary of $[16 ;$ p. 348$]$, we deduce

2.5.1. If $G$ is a locally solvable group and $1 \neq x \in G$, then $\left(x^{G}\right)^{\prime}<$ $x^{G}$.

III. The Structure of Locally Finite Groups in $I \Re(s)-\Re(s)$. W need several lemmas before proceeding with the main result.

LEMMA 3.1. $\Re(s) \cap L \mathscr{F}=(L \Re) \cap L \mathfrak{F}$.

Proof. We need only note that $L \Re \subset \Re(s)[14 ;$ p. 222]

LemMA 3.2. If $G \in I \Re(s) \cap L \mathscr{\mho}, \Sigma$ is a radical class, and $H$ is maximal $\Sigma$ subgroup with infinite normalizer, then $H \triangleleft G$.

Proof. From the hypotheses, $H$ ser $G$. Now use 2.1.2.

LEMMA 3.3. If $G \in I \mathfrak{N}(s) \cap L \mathscr{\mho}$ and for some finite normal subgrou $F, G / F \in \mathfrak{R}(s)$, then $G \in \mathfrak{\Re}(s)$.

Proof. By Lemma 3.1, it suffices to show that $G \in L \Re$. We do th by showing that all Sylow $p$ subgroups are normal. In view of Lemm 3.2 , we need only show that finite Sylow $p$ subgroups have infini normalizers. Let $K$ be a finite Sylow $p$-subgroup of $G$. Then $K F / F$ is Sylow $p$ subgroup of $G / F$ and hence $K F \triangleleft G$. This implies that $N(1$ has finite index in $G$. By Lemma 3.2, $K \triangleleft G$. 
LemMA 3.4. If $G \in I \mathfrak{N}(s) \cap L \mathscr{F}$ and some collection $\Gamma \neq \varnothing$ of infinite normal subgroups has the property $\cap \Gamma=1$, then $G \in \Re(s)$.

Proof. Let $x$ and $y$ be elements of coprime order in $G$, then (using Lemma 3.1) $[x, y] \in H$ for each $H \in \Gamma$. Thus $[x, y] \in \cap \Gamma=1$ so that $x$ and $y$ commute. It follows that $G \in L \Re$.

LEMMA 3.5. If $G \in I \Re(s) \cap L \mathscr{z}$ and $G=P] R(]=$ split extension), where $P$ is a $p$ group and $R$ is a finite $p^{\prime}$ group, then for all infinite subgroups $Y \leqq P$ with $Y \triangleleft G$, we have $Y R \triangleleft G$ and $[P, R] \leqq Y$.

Proof. By Lemma 2.4.2, YR/Y is a Sylow $p^{\prime}$ subgroup of G/Y. Since, $G / Y \in \Re(s) \cap L \mathscr{Z} \leqq L \Re$, both results follow.

TheOREM A. If $G \in(I \Re(s)-\Re(s)) \cap L \mathscr{Z}$, then $G$ has an infinite Sylow $p$ subgroup $P$ such that $G=P] K$, where $N(K)$ is a finite group and $K$ is a non-identity nilpotent group.

Proof. Let $A$ be the intersection of all the infinite normal subgroups of $G$. $A$ is infinite by Lemma 3.3 and 3.4. Thus $A$ has an infinite abelian subgroup $Y([10]$ or $[18 ; p .95])$. It follows that $A=Y^{G}$ is $L \Re$ (2.1.2). Thus, $A=\Pi\left\{Q_{i} \mid i \in I\right\}$, where $Q_{i}$ is a non-identity Sylow $q_{i}$ subgroup of $A$. For each $i, Q_{i} \triangleleft G$. Thus, if $|I|>1$, all $Q_{i}$ 's are finite, and since $A$ is infinite, we conclude that $|I|$ is infinite. But this yields a proper infinite subgroup of $A$ that is normal in $G$. Hence $|I|=$ 1 , and $A$ is a $p$ group.

Let $P$ be a Sylow $p$-subgroup of $G$ containing $A$; since $P$ is infinite, we have $P \triangleleft G$. We now show that $G / P$ is finite. Suppose that $G / P$ is infinite. Let $x$ and $y$ be $p^{\prime}$ elements of $G$ and $U \leqq G$ such that $x, y \in$ $U$ and $U / P$ is countably infinite. Let $T$ be a transversal of $P$ in $U$ and $T_{1}=\langle T\rangle$. Now $P \cap T_{1}$ is a normal Sylow $p$ subgroup of $T_{1}$, and $T_{1}$ is countable. By 2.4.1, there is a $p^{\prime}$ group $Q \leqq T_{1}$ such that $T_{1}=(P \cap$ $\left.T_{1}\right) Q$. Then $U=T_{1} P=Q\left(P \cap T_{1}\right) P=Q P$. Evidently, $Q$ is an infinite $p^{\prime}$ subgroup of $U$. By 2.1.2, $Q^{U}$ is a $p^{\prime}$ group. Thus $U=Q^{U} \times P$ and $Q^{U}$ is a normal Sylow $p^{\prime}$ subgroup of $U$. Thus, $x, y \in Q^{U}$, so that $\langle x, y\rangle$ is a $p^{\prime}$ group. We conclude that $S=\{x \in G \mid(|x|, p)=1\}$ is a $p^{\prime}$ subgroup of $G$. $S$ is clearly infinite. Lemma 3.2 now yields $S \triangleleft G$, and consequently we have $G=P \times S$. This contradicts Lemma 3.4. Thus, $G / P$ is finite. Further, since $P$ is infinite, $G / P \mathfrak{R}(s)$; hence, $G / P$ is nilpotent.

We are now permitted to apply 2.4.2 to produce a complement $K$ of $P$. If $N(K)$ were infinite, we would have $K \triangleleft G$ by Lemma 3.2; this implies $G=P K \in L \Re$, a contradiction.

THEOREM B. If $G \in(I \Re(s)-\mathfrak{N}(s)) \cap L \mathscr{F}$, then $G$ is a Černikov group. 
Proof. Write $G=P] K$, where $P$ and $K$ are as in Theorem $A$, a let $A$ be the intersection of the infinite normal subgroups of $G$. Th $A \leqq P$ and $A$ is infinite (Lemma 3.4). We show that $A^{\prime}$ is finite. If is infinite, then $A=A^{\prime}$. Since $G$ is locally solvable, $x \in A$ impl $x^{G}<A(2.5 .1)$. Thus for each $x \in A, x^{G}$ is finite, and $G / C\left(x^{G}\right)$ is fini It follows that $A /\left(A \cap C\left(x^{G}\right)\right)$ is finite and this implies that $A \leqq C(x$ Hence $A^{\prime}=1$, a contradiction. Thus $A^{\prime}$ is finite.

Let $G_{1}=G / A^{\prime}$ and $A_{1}=A / A^{\prime}$. By Lemma 3.3, $G_{1} \in(I \Re(s)$ $\mathfrak{R}(s)) \cap L \mathscr{F}$; further $A_{1}$ contains no proper normal infinite subgrou of $G_{1}$. Now $A_{1}(p) \triangleleft G_{1}$. If $A_{1}(p)$ is infinite, then $A_{1}(p)=A_{1}$. Th $A_{1}$ is residually finite and consequently $A_{1} K_{1}$ is residually finite, whe $K_{1}=K A^{\prime} / A^{\prime}$. By Lemma 3.4, $A_{1} K_{1} \in \mathfrak{N}(s)$. Thus $A_{1} \leqq C\left(K_{1}\right)$ and Lemma 3.2, $K_{1} \triangleleft G_{1}$; also $K_{1}$ is a nilpotent $p^{\prime}$ group and $G_{1}=P / A^{\prime} \times$ Thus $G_{1}$, as a direct product of two $L \mathfrak{R}$ groups, is in $L \mathfrak{N} \cap L \mathfrak{F}$ $\mathfrak{N}(s)$, a contradiction. We conclude that $A_{1}(p)$ is finite.

Since $A_{1}(p)$ is finite $A_{1}$ and consequently $A$, has min. Further, $C(A$ $\leqq C(K)$. Note that $K$ is a Sylow $p^{\prime}$ subgroup of $G(2.4 .2)$, and that $K$ nilpotent (Theorem A). Suppose for the moment that $N(K)$ is infini Lemma 3.2 then forces $K \triangleleft G$. At this stage, we have $G=P \times$ Then $G$, as the direct product of two $L \Re$ groups, is $L \Re$. This viola the assumption that $G \notin \Re(s)$. We conclude then that $N(K)$, and her $C(K)$, is finite. The finiteness of $C(K)$ implies that $C(A K)$ is finite. Lemma 3.5, $N(A K)=G$. By 2.2.1, $G / C(A K)$ is a Černikov group. No $G$ is an extension of a finite group by a Cernikov group. It now follo from 2.2.4 that $G$ is a Cernikov group.

TheOREM C. Let $G \in(I \mathfrak{N}(s)-\Re(s)) \cap L \mathfrak{\mho}$ and write $G=P] K$ in Theorem $A$. If $x \in K$ normalizes some proper infinite subgroup $d(P)$, then $x \in C(P)$.

Proof. Let $x \in K$, and $H$ be an infinite proper subgroup of $d$ such that $H^{\langle x\rangle}=H$. We now show that $C(x) \cap d(P)$ is infinite.

If $C(x) \cap d(P)$ is finite, then $[d(P),\langle x\rangle]$ has finite index in $d(P)$ 2.2.2. But we also have $[d(P),\langle x\rangle]<H$ by Lemma 3.5 (applied to $I \Re(s)$ group $d(P)\langle x\rangle)$. Since $H$ has infinite index in $d(P)$, we hav contradiction. Hence, $C(x)$ is infinite and by Lemma 3.2, we ha $\langle x\rangle \triangleleft P\langle x\rangle$. Thus $x \in C(P)$.

THEOREM D. An infinite locally finite group $G$ is in $I \mathfrak{N}(s)-\mathfrak{N}(s$ and only if $G$ has an infinite normal Sylow $p$ subgroup $P$ such that

(i) P is a Černikov p group,

(ii) $G=P] K$ where $K \neq 1$, and $G / d(P)$ is a finite nilpotent group,

(iii) if $x \in K$ normalizes an infinite proper subgroup of $d(P)$, th $x \in C(P)$,

(iv) there is an $x \in K$ such that $[x, P] \neq 1$. 
Proof. The necessity of conditions (i)-(iv) is incorporated in Theorems A-C. We show here that in any group $G$ with properties (i)-(iv), every infinite subgroup is ascendant.

Let $H$ be an infinite subgroup of $G$. If $d(P) \leqq H$, then $H$ sn $G$ since $G / d(P)$ is nilpotent. Suppose $d(P) \neq H$. Then $H=(H \cap P)] L$, $H \cap P$ being a normal Sylow $p$ subgroup of $H$ and $L$ a $p^{\prime}$ group (2.4.2). By 2.4.2, $L$ is conjugate to a subgroup $L^{t}$ of $K$.

Now, $H^{t} \cap d(P)$ is a proper infinite subgroup of $d(P)$ and is normalized by $L^{t}$. By condition (iii) $L^{t} \leqq C(P)$ and so $L \leqq C(P)$. We now have $H=(H \cap P) \times L$ asc $P \times L(2.2 .3)$. Since $P \times L$ sn $G$, we have $H$ asc $G$.

The condition (iv) insures that $G \notin \mathfrak{R}(s)$.

Later in $\$ I V$, we give a somewhat different statement of Theorem D (Theorem $\mathrm{D}^{\prime}$ ). To do this, we shall need

Corollary 3.1. Let $G \in(I \Re(s)-\Re(s)) \cap L \mathscr{8}$. Then, in the notation of Theorem $\mathrm{D}, d(P)=[P, K]$ and $[d(P), K]=d(P)$.

Proof. It suffices to prove $[d(P), K]=d(P)$. Suppose $[d(P), K]<$ $d(P)$. Using 2.2.2, we see that $C_{d(P)}(K)$ is infinite, as $d(P) /[d(P), K]$ is infinite. Then, Theorem $\mathrm{D}$ (iii) yields $K \leqq C(P)$ which violates part (iv) of Theorem D. Thus, $[d(P), K]=d(P)$.

As a consequence of Theorem $\mathrm{D}$, we have

Conollary 3.2. $(\operatorname{IN}(s)-\mathfrak{N}(s)) \cap L \mathscr{F}=(\operatorname{IN}(a)-\mathfrak{N}(a)) \cap L \mathfrak{F}$.

Proof. Suppose $G \in(I \Re(s)-\Re(s)) \cap L \mathfrak{z}$. By Theorem $D, G$ has min. This implies that $G \in I \mathfrak{N}(a)$. Evidently, $G \notin \mathfrak{R}(a)$. This yields $(I \mathfrak{R}(s)-\mathfrak{R}(s)) \cap L \mathfrak{F} \leqq(I \mathfrak{R}(a)-\mathfrak{R}(a)) \cap L \mathfrak{F}$. For the other inclusion, let $G \in(I \mathfrak{N}(a)-\mathfrak{N}(a)) \cap L \mathscr{\mho}$. Clearly $G \in I \mathfrak{N}(s)$. Further it is not hard to show the existence of a finite subgroup $F$ of $G$ with $N(F)=F$. In view of 2.1.1, $F$ is not a serial subgroup. This establishes $(I \mathfrak{R}(a)-$ $\mathfrak{R}(a)) \cap L \mathscr{F} \leqq(I \mathfrak{R}(s)-\mathfrak{R}(s)) \cap L \mathscr{f}$ and the corollary is proved.

Also of interest is

CoRollary 3.3. An uncountable $\operatorname{IN}(s) \cap L \mathfrak{F}(\operatorname{IN}(a) \cap L \mathscr{F})$ group is an $\mathfrak{R}(s)(\mathfrak{R}(a))$ group.

Corollary 3.3 is proved by observing that the groups occurring in Theorem $\mathrm{D}$ are countable.

IV. Strongly Irreducible Automorphisms of Divisible Abelian Groups. 
Definition. Let $P$ be a divisible abelian $p$ group of finite rank (i. the $p$-rank of the abelian group $P,[19 ;$ p. 35]) and $A$ a periodic gro of automorphisms of $P$. A is called strongly irreducible $(S-I)$ if each $1 \neq a \in A$, and each infinite subgroup $H \leqq P, H^{\langle\alpha\rangle}=P$.

These are the types of automorphisms that arise in Theorem D (i In the section, we prove

THEOREM E. Let $P$ be a divisible abelian p-group of finite rank, a $A$ be a nilpotent $p^{\prime}$ group of $S-I$ automorphisms of $P$. Then

(i) A is cyclic,

(ii) if $1 \neq a \in A$, then $\langle a\rangle$ acts irreducibly on $P(p)$,

(iii) if $n=\operatorname{rank}(P)$, and $q$ is the smallest prime dividing $|A|$, th $n=$ the order of $p(\bmod q)$; and so $n \mid q-1$.

Theorem $\mathrm{E}$ will be used to give a different formulation of Theore D. Before proceeding further, we note that periodic groups of aut morphisms of divisible abelian $p$ groups of finite rank are finite [ ] p. 84]. Thus, we assume all $S-I$ groups of automorphisms are fini Also, throughout the remainder of this section, $P$ will denote a divisil abelian $p$ group of finite rank.

LeMmA 4.1. Let $1 \neq A$ be an $S-I$ subgroup of $\operatorname{Aut}(P) w$ $(|A|, p)=1$. Then for each element $y \in P(p), y \neq 1$, and each $e$ ment $1=a \in A$, we have $y^{\langle\alpha\rangle}=P(p)$.

Proof. Let $1 \neq a \in A$. We view $P$ as a module for the ring $R$ $Z\langle a\rangle$. By 2.3.1 $P$ is an injective $R$ module, and it is not difficult to shi that $P$ is an $R$ injective hull of $P(p)$.

Let $1 \neq y \in P(p)$ and $H=y^{\langle\alpha\rangle}$. Then $H$ is an $R$ submodule of If $H<P(p)$, Maschke's theorem yields a $R$ submodule $0 \neq H_{1} \leqq P$ such that $P(p)=H \oplus H_{1}$. We now use 2.3.2 to obtain $P=\overline{P(p)}=$ $\oplus \overline{H_{1}}$; here the ,s denote $R$ injective hulls. Now $\bar{H}$ is an infinite invariant subgroup of $P$ so that $\bar{H}=P$. This forces $\overline{H_{1}}=0$ and $c($ sequently $H_{1}=0$ (in the module notation). This contradicts the sumption $H<P(p)$. Thus $H=P(p)$ and the proof is complete.

The following easy remark will simplify some of our arguments.

LeMma 4.2. Let $G$ be any abelian $p$ group and $\alpha \in \operatorname{Aut}(G)$ with finite and $(|\alpha|, p)=1$. If $\alpha$ centralizes all elements of order $p$ in then $\alpha=1$.

Proof. Suppose the lemma false and let $x \in G$ be of least order st that $\alpha \notin C(x)$. Since $G$ is abelian, $[x, \alpha]^{p}=\left[x^{p}, \alpha\right]$. By the minimal of $|x|,\left[x^{p}, \alpha\right]=1$. Thus $[x, \alpha]^{p}=1$ and $[x, \alpha] \in C(\alpha)$. Thus, 1 $[x, \alpha]^{p}=\left[x, \alpha^{p}\right]$. Therefore $\left\langle\alpha^{p}\right\rangle=\langle\alpha\rangle \leqq C(x)$; a contradiction. 
LEMMA 4.3. Let $1 \neq A$ be an $S-I$ subgroup of $\operatorname{Aut}(P)$ with $(|A|, p)=1$. Let $n=\operatorname{rank}(P)$, and $\alpha: A \rightarrow G L(n, p)$ be defined by $a \alpha=a \mid P(p)$ (the restriction of a to $P(p)$ ). Then $\alpha$ is an isomorphism and for each $1 \neq a \in A,\langle a \alpha\rangle$ is an irreducible subgroup of $G L(n, p)$.

Proof. Certainly $\alpha$ is a homomorphism. Lemma 4.2 implies ker $\alpha=$ 1. The irreducibility of $\langle a \alpha\rangle$ follows from Lemma 4.1.

Proof of Theorem E. Lemma 4.3 immediately gives (ii). Let $T$ be an abelian subgroup of $A$. By Lemma 4.3, $T$ is an irreducible subgroup of $G L(n, p)$. As is well known, abelian irreducible subgroups of $G L(n, p)$ are cyclic $[8 ; p .65]$. Thus $T$ is cyclic. Thus, if $q$ is a prime, $q \neq 2$, then the Sylow $q$ subgroup of $A$ is cyclic [20; p. 252]. Let $L$ be the Sylow 2 subgroup of $A$, and $s \in L$ be an element of order 2. Since $\langle s\rangle$ acts irreducibly on $P(p)$, we must have $r(P)=1$. Thus, (again by Lemma 4.3) $L$ is cyclic.

Hence, all Sylow subgroups of $A$ are cyclic. The nilpotence of $A$ implies now that $A$ is cyclic. This establishes (i).

Let $q$ be the smallest prime divisor of $A$ and $a \in A$ be an element of order $q$. Then $P(p)$ is a faithful and irreducible module for $\langle a\rangle$; i.e. $P(p)$ is irreducible as a $Z_{p}\langle a\rangle$ module. The possible dimensions of such modules are enumerated in the book [13; II, 3.10]. In particular, we must have $n=\operatorname{order}$ of $p(\bmod q)$. Consequently, $n \mid q-1$. This completes the proof of Theorem $\mathrm{E}$.

The proof of Theorem E shows than an $S-I$ subgroup $A$ of Aut $(P)$ has cyclic Sylow $q$ subgroups for $q \neq p$. Cernikov has shown [2; p. 723-724] that the Sylow $p$ subgroups of $A$ are also cyclic. The proof of this fact makes essential use of results in the paper [5]

In view of Theorem $\mathrm{E}$ and Corollary 1 of Theorem $\mathrm{D}$, we may now give a somewhat different form of Theorem $D$.

Theorem $\mathrm{D}^{\prime} . \quad G \in(I \mathfrak{N}(s)-\mathfrak{R}(s)) \cap L \mathfrak{F}$ if and only if $G$ has an infinite normal Sylow $p$ subgroup $P$ such that

(i)' $P$ is a C'ernikov $p$ group,

(ii) ' $G=P] K$ where $K$ is a finite nilpotent $p^{\prime}$ group and $K \neq 1$,

(iii) ' $Y=[P, K]$ has finite index in $P$ and is a divisible abelian group with min,

(iv) ' $K / C_{C}(P)$ is a non-trivial cyclic group of $S-I$ automorphisms of $Y$.

We note the following special case of this theorem.

Corollary 4.1. Suppose in the notation of Theorem $D^{\prime}$ that $Y=P$ (i.e., $P$ is divisible abelian of finite rank). Let $H$ be an infinite subgroup 
of $G$, where $G$ satisfies the conditions of Theorem $D^{\prime}$. Then either

(a) $P \leqq H$,or

(b) $H=(H \cap P) \times L$ where $L$ is conjugate to a subgroup of $K$. Consequently, $H$ sn $G$.

If, in addition, $C_{K}(P)=1$, then $H \mathrm{sn}_{2} G\left(H \mathrm{sn}_{2} G\right.$ means $\left.H \triangleleft H^{G}\right)$.

This corollary can be proved by using Theorem $\mathrm{E}$ in conjunction with the method of proof of Theorem $\mathrm{D}$. We do not repeat these arguments here.

V. Construction of Groups Satisfying the Conditions of Theorem D. This task turns out to be rather easy if we make use of the results of 2.3 .

Theorem F. Let $E$ be an elementary abelian $p$ group of rank $n$. Further, let A be a cyclic $p^{\prime}$ subgroup of $G L(n, p)$ such that every nonidentity subgroup of A acts irreducibly on E. Viewing $E$ as an $R=$ ZA module, let $\bar{E}$ be an R-injective hull of $E$. Then $\bar{E}$ is a divisible abelian p-group of rank $n$ and the group $G=\bar{E}$ ] A satisfies the conditions of Theorem $D$ (or Theorem $D^{\prime}$ ). (Here the action of $A$ on $\bar{E}$ is induced from the action of $R$ on $\bar{E}$ ).

Proof. The existence of $R$ injective hulls is well known [6; p. 389]. We first note that $\bar{E}$, as an abelian group, is divisible. This is a consequence of $[6 ;$, p. 393, Ex. 3]. Let $Y$ be the Sylow $p$-subgroup of $\bar{E}$. Then $Y$ is a divisible abelian group and is also on $R$-submodule of $\bar{E}$. Now 2.3.1 implies that $Y$ is injective as an $R$-module. Since $E \leqq Y$, we deduce that $Y=\bar{E}$; thus $\bar{E}$ is a $p$-group.

Suppose $\bar{E}(p)>E$, and let $y \in \bar{E}(p)-E$. Then by Maschke's theorem, $D=E+y R=E \oplus F$ for some $R$ submodule $F$ of $D$ ( $y R$ is a finite elementary $p$-group). On the other hand, $F \cap E \neq 0$, since $\bar{E}$ is an essential extension of $E$. This contradiction shows that $\bar{E}(p)=E$; thus, $\bar{E}$ has rank $n$.

Let $1 \neq a \in A$ and suppose $H$ is an infinite $\langle a\rangle$ invariant subgroup of $G$. Then $d(H)$ is an infinite divisible group and $d(H)$ is $\langle a\rangle$ invariant. Certainly $d(H) \cap E \neq 1$; this follows from the fact that $\operatorname{rank}(E)=\operatorname{rank}(\vec{E})$. The $\langle a\rangle-$ irreducibility of $E$ now yields $E \leqq$ $d(\mathrm{H})$. Thus, $\operatorname{rank}(d(H))=\operatorname{rank}(\bar{E})=n$. The divisibility of $d(H)$ now implies that $d(H)=\bar{E}$ (otherwise $d(H)$ would be a proper direct summand of $\bar{E}$ ). Thus, $H=\bar{E}$ and $A$ is an $S-I$ group of automorphisms of $\bar{E}$.

It is now a simple matter to show that all the conditions of Theorem $D$ hold. This completes the proof of Theorem F. 
We note some additional properties of the group $G$ constructed in Theorem F.

$5.1[\bar{E}, A]=\bar{E}$.

5.2 If $H$ is an infinite subgroup of $G$, then either

(a) $\bar{E}<H$, or

(b) $H$ is abelian and $H \mathrm{sn}_{2} G$.

5.3 Every non-abelian infinite subgroup is normal.

The property 5.1 follows from Corollary 3.1 , while 5.2 and 5.3 are consequences of Corollary 4.1.

VI. Non-periodic $I \Re(a)$ Groups. The main result here is that an element of infinite order in an $I \mathfrak{N}(a)$ group forces the group to be an $\mathfrak{R}(a)$ group. Similar results hold for the class $I \mathfrak{R}(s n)$. However, we have been unable to develop a corresponding theory for either of the classes $I \Re(s)$ or $I \Re(d)$. The key fact that enables us to prove our main result here is the local nilpotence of groups in the class $\Re(a)$ ([18; p. 61 ). Whether or not $\mathfrak{R}(s)$ groups (or even $\mathfrak{N}(d)$ ) groups are locally nilpotent is an unresolved question.

THEOREM $G$ [3; p. 29]. If $G$ is not periodic and $G \in \operatorname{I\Re (}(a)(\operatorname{IN}(\mathrm{sn}))$, then $G \in \mathfrak{N}(a)(\mathfrak{N}(\mathrm{sn}))$.

Proof. Let $x$ be an element of infinite order in the $I \Re(a)$ group $G$ and let $F$ be a finite subgroup of $G$. Then $\langle x\rangle$ asc $\langle F, x\rangle$ so that $x^{F} \in$ $L \Re\left([9]\right.$ or $\left[18 ;\right.$ p. 61]). The finiteness of $F$ insures that $x^{F}$ is finitely generated. Thus, $x^{F}$ is a finitely generated nilpotent group and consequently $D$, the center of $x^{F}$ is a finitely generated infinite abelian group. $D$ contains an infinite characteristic torsion free subgroup $E$. Let $S=E F$ and $p$ be a prime not dividing $|F|$. The group $S / E^{p^{n}}$ is a finite $\mathfrak{R}(a)$ (and hence nilpotent) group for each positive integer $n$. Thus, for each $n,[E, F] \leqq E^{p^{n}}$, and this gives $[E, F] \leqq \cap E^{p^{n}}=1$.

We now have $F \triangleleft E F=S$ asc $G$ which completes the proof. The same proof shows that non-periodic $I \mathfrak{N}(\mathrm{sn})$ groups are $\mathfrak{R}(\mathrm{sn})$.

\section{Biblography}

1. R. Baer, Finite extensions of abelian groups with minimum condition, Trans. Amer. Math. Soc. 79 (1955), 521-540.

2. S. N. Cernikov, Groups with given properties of systems of infinite subgroups, Ukrain. Mat. Z. 19 (1967), 111-131; Ukranian Math. J. 19 (1967), 715-731.

3. - On the normalizer condition, Math. Zametki 3 (1968), 45-50; Math. Notes (USSR) 3 (1968), 28-30.

4. __ On locally solvable groups satisfying the minimal condition for subgroups, Mat. Sb. 28 (70) (1951), 119-129 (Russian). 
5. _- On infinite special groups with finite center, Mat. Sb. 17 (1945), 105-130 (Russian).

6. C. W. Curtis and I. Reiner, Representation theory of finite groups and associative algebras, Interscience, New York (1962).

7. O. H. Kegel and B. A. F. Wehrfritz, Locally finite groups, North-Holland, New York (1973).

8. D. Gorenstein, Finite groups, Harper and Row, New York (1968).

9. K. Gruenberg, The Engel elements of a soluble group, Illinois J. Math. 3 (1959), 151-168.

10. P. Hall and C. R. Kulatilaka, A property of locally finite groups, J. London Math. Soc. 39 (1964), 235-239.

11. B. Hartley, Serial subgroups of locally finite groups, Proc. Cambridge Philos. Soc. 71 (1972), 199-201.

12. B. Hartley and D. McDougall, Injective modules and soluble groups satisfying the minimal condition for normal subgroups, Bull. Austral. Math. Soc. 4 (1971), 113-135.

13. B. Huppert, Endliche Gruppen I, Springer-Verlag, New York (1967).

14. A. G. Kurǒs, Theory of groups, Vol. 2, Chelsea, New York (1960).

15. A. G. Kurǒs and S. N. Černikov, Soluble and nilpotent groups, Uspehi Mat. Nauk. 2 (1947), 18-59; Amer. Math. Soc. Translations 80 (1953).

16. R. E. Phillips and K. K. Hickin, On classes of groups defined by systems of subgroups, Archiv Der Math. 24 (1973), 346-350.

17. B. I. Plotkin, Radical groups, Mat. Sb. 37 (79) (1955), 507-526; Amer. Math. Soc. Translations (2), 17, 9-28.

18. D. J. S. Robinson, Finiteness conditions and generalized soluble groups, part 1, Springer-Verlag, New York (1972).

19. - Finiteness conditions and generalized soluble groups, part 2, Springer-Verlag, New York (1972).

20. W. R. Scott, Group theory, Prentice-Hall, Englewood Cliffs, New Jersey (1964).

Michigan State University, East Lansing, Michigan 48823 